\title{
La formación en la empresa: ¿cómo se mide?
}

\author{
Cecilia Albert \\ Carlos García-Serrano \\ Virginia Hernanz \\ (Universidad de Alcalá)
}

\section{Resumen}

Este trabajo plantea la utilización de tres fuentes estadísticas alternativas (y complementarias) para medir la proporción de trabajadores que realizan actividades de formación en la empresa, discute sus ventajas e inconvenientes, e investiga el perfil de los trabajadores que realizan formación. De forma adicional, se analiza la intensidad de la formación medida por el tiempo que los trabajadores dedican a la misma. Los resultados sugieren que es más probable que un trabajador participe en actividades de formación cuanto mayor es su nivel de estudios, cuanto más cualificada es la ocupación en la que trabaja y cuanto más grande es la empresa para la que lo hace. También se ha encontrado una cierta evidencia de que la intensidad de la formación (el tiempo que se dedica a ella) es mayor entre los trabajadores con contrato temporal y con menor antigüedad en el puesto de trabajo.

Palabras clave: formación en la empresa

Clasificación JEL: J24, M53

\begin{abstract}
In this paper we use three alternative (and complementary) data sources to measure the proportion of workers who paticipate in on-the job training, we discuss its advantages and disadvantages and investigate the profile of workers who carried out the training. In addition, we analyze the intensity of the training measured by the time spent by workers. The results suggest that it is more likely that a worker takes part in training activities the higher is his/her education level, the more qualified is his/her occupation and the larger the size of the firm. We have also found evidence that the intensity of training (time spent on it) is higher among workers with temporary contracts and less tenure in his job.
\end{abstract}

Keywords: on-the-job training

JEL Classification: J24, M53

Agradecimientos: Los autores agradecen la financiación recibida de la Universidad de Alcalá a través del proyecto de investigación UAH2004/022 titulado «Los efectos de la formación continua sobre los salarios: un estudio comparativo entre los países de la Unión Europea».

\section{Introducción}

Desde diferentes instancias se considera crucial que los trabajadores participen en actividades formativas. Desde un punto de vista de política, la formación en la empresa (o continua) constituye un elemento fundamental para mejorar los resultados eco- 
nómicos, puesto que se considera que contribuye al crecimiento de la productividad de un país. Desde un punto de vista individual, la formación también es relevante ya que se estima que es un determinante importante de los salarios y de la productividad de los trabajadores.

Los determinantes de quiénes reciben formación en las empresas están relacionados con los costes y los rendimientos de esta inversión en capital humano. La selección de los trabajadores que participan en actividades formativas va a estar condicionada por el objetivo de asegurar que los trabajadores que la reciben van a generar los mayores aumentos de la productividad para su empresa durante el mayor tiempo posible. Esto significa que determinadas características personales pueden estar relacionadas con la participación en actividades formativas pero también lo estarán otras vinculadas al puesto de trabajo y a la empresa en la que está ocupado el trabajador.

Uno de los objetivos de este artículo es ofrecer un análisis de las características de los trabajadores que realizan formación en las empresas españolas. Este análisis permitirá identificar los colectivos que reciben más formación y los que menos, lo cual es importante ya que las diferencias en la provisión de formación entre trabajadores y empresas puede ser una fuente relevante de desigualdad no sólo en las remuneraciones salariales sino también en otras variables laborales (como la estabilidad en el empleo o la productividad).

Sin embargo, hay un problema fundamental previo para el estudio de la formación en el empleo: su medición. Y es que cada fuente estadística tiene una forma diferente de preguntar a los individuos sobre las actividades formativas que han realizado y sobre su duración. También suele ser diferente el periodo temporal al que se refieren las preguntas sobre formación. Todo ello genera medidas distintas de la incidencia de la formación. Por ello, un aspecto novedoso de este artículo es que no se utiliza una sola fuente de datos para abordar la medición de la formación en la empresa y las características de los trabajadores que la reciben sino que se utilizan tres: la Encuesta de Población Activa (EPA), la Encuesta de Calidad de Vida en el Trabajo (ECVT) y el Panel de Hogares de la Unión Europea (PHOGUE) ${ }^{1}$. Esto implica, por un lado, estudiar y comparar las distintas medidas de formación en la empresa que pueden construirse a partir de cada una de estas encuestas; y por otro, examinar las características de los trabajadores que reciben esta formación, con el propósito de determinar si independientemente de la fuente de información y de la manera de medir la formación en la empresa, existen o no características que influyen en la probabilidad de que un trabajador realice o no actividades de formación. De forma adicional, también se investiga (por primera vez y de forma exploratoria) cuál es la duración de

\footnotetext{
Estas encuestas van dirigidas a los trabajadores, es decir, son los empleados los que aportan información sobre su formación. Es importante tener presente este hecho dado que se ha comprobado que existe una disparidad entre empleados y empleadores en las respuestas a idénticas preguntas sobre formación en la empresas (véase Barron et al., 1997). En este sentido, podrían compararse las cifras de las bases de datos que se utilizan en este trabajo y las que proporciona, por ejemplo, la Encuesta de Formación Profesional Continua, realizada en 1993 y 1999.
} 
las actividades formativas en la empresa y si existen características de los trabajadores asociadas a duraciones más largas o más cortas.

Los trabajos que se han ocupado de cuestiones relacionadas con la formación son numerosos ${ }^{2}$. En el caso español, existen algunos trabajos que han abordado el estudio de los determinantes de la formación en la empresa: Alba (1994), con datos de empresas provenientes de la «Encuesta de Negociación Colectiva en las Grandes Empresas» (ENCGE) de 1989; Peraita $(2000,2005)$, con la primera ola del PHOGUE; Alba y Tugores (2000), que utilizan la primera ola del PHOGUE y la EPA para el periodo 1987-1998; y Abellán y Felgueroso (2005), con datos de la ECVT del 2001. Otros estudios se han ocupado además de la relación entre temporalidad y formación (Caparrós et al., 2004, y Albert et al., 2005) y del efecto de la formación en los salarios (Albert et al., 2007).

La estructura del artículo es la siguiente. La sección dos aborda las cuestiones relacionadas con la medición de la formación en la empresa, presentando las bases de datos que pueden utilizarse para realizar dicha medición en el caso de la economía española, mostrando las similitudes y las diferencias de las preguntas que emplean para obtener la información sobre formación y discutiendo los resultados obtenidos. En la sección tres, se analiza la participación de los trabajadores en las actividades formativas de las empresas y la influencia de distintas características de los individuos, los puestos de trabajo y las empresas en la probabilidad de que los trabajadores realicen dicha formación. La sección cuatro se centra en la intensidad de las actividades formativas aproximándola por el tiempo que el trabajador dedica a dicha formación. Finalmente, la sección cinco ofrece un resumen y las conclusiones del trabajo.

\section{La medición de la formación en la empresa}

En este trabajo se van a utilizar tres bases de datos que proporcionan información sobre la formación en la empresa: la Encuesta de Población Activa (EPA), la Encuesta de Calidad de Vida en el Trabajo (ECVT) y el Panel de Hogares de la Unión Europea (PHOGUE). En esta sección, se presentan las preguntas de los cuestionarios de estas tres fuentes estadísticas referidas a formación y se explica cómo se construyen las variables que sirven para medir la participación en actividades formativas a partir de las respuestas de los individuos a dichas preguntas.

Comenzamos por la EPA. El cuestionario de esta encuesta permite disponer de una gran cantidad de información sobre características socio-económicas que tienen que ver con la actividad económica, el empleo, el paro, la inactividad, etc. Además, contiene varias preguntas referidas a la formación ${ }^{3}$. En primer lugar, se interroga a todos

2 Entre los trabajos teóricos destacan Becker (1962), Katz y Ziderman (1990), Altonji y Spletzer (1991), Stevens (1994) y Acemoglu y Pischke (1998, 1999), y entre los empíricos Greenhalgh y Stewart (1987), Lynch (1991, 1992), Royalty (1996), Loewenstein y Spletzer (1999) y Booth et al. (2003).

3 Aquí se presentan las preguntas referidas al cuestionario del periodo 1999-2004, que son bastante similares a las del periodo 1992-1998 aunque han cambiado en algunos aspectos en el cuestiona- 
los individuos sobre si han seguido durante las últimas cuatro semanas algún tipo de estudios o de formación: las posibles respuestas son «sí», «estudiantes en vacaciones» o «no». En segundo lugar, quienes contestan las dos primeras respuestas deben responder a otras cinco preguntas sobre formación: qué tipo de estudios o formación han seguido y en qué sector, cómo han realizado esos estudios, cuál era el principal objetivo de dicha enseñanza, cuál fue la duración total de la formación y cuál es el número habitual de horas de formación a la semana.

A partir de la información suministrada por los individuos, es posible construir una variable dicotómica relativa a la formación en la empresa combinando dos de dichas preguntas. Esta variable toma el valor uno si los individuos dicen «sí» cuando se les pregunta si han seguido durante las últimas cuatro semanas algún tipo de estudios o de formación y, además, indican que han seguido dichos estudios o formación «haciendo prácticas en una empresa» o en un «sistema mixto (asistiendo a un aula y haciendo prácticas en una empresa)» ${ }^{4}$.

La ECVT es una investigación muestral de carácter anual que se realiza por el Ministerio de Trabajo y Asuntos Sociales (MTAS) por primera vez en 1999. Se trata de una encuesta dirigida a los individuos ocupados mayores de 16 años que residen en viviendas familiares de todo el territorio nacional, con excepción de Ceuta y Melilla. Estar ocupado significa haber estado trabajando al menos una hora durante la semana de referencia (la anterior a la de la entrevista). El diseño de la muestra tiene en cuenta tres variables: la comunidad autónoma de residencia, el tamaño del municipio y el número de habitantes en la sección censal.

En esta encuesta, se dispone de información sobre características individuales de los trabajadores (sexo, edad, estado civil, hijos y nivel de estudios) y características de la empresa en que trabajan y del puesto de trabajo que ocupan (sector institucional, rama de actividad económica, grupo de ocupación, tamaño de la empresa, jornada laboral, antigüedad y tipo de contrato).

Además, la ECVT proporciona información sobre si la empresa en la que trabaja el individuo financia u organiza formación para los empleados y si el trabajador en cuestión ha participado o no en dicha formación. En particular, se efectúa a los trabajadores la siguiente pregunta: «Durante el último año, ¿ha realizado su empresa alguna actividad de formación para sus empleados?» (V184). Además, a aquellos que responden que sí a esta pregunta se les interroga sobre:

- la frecuencia con que participaron en cursos de formación continua realizados por su empresa (V186) (esto sucedía hasta el año 2001);

rio de 2005 (por ejemplo, se ha introducido una nueva pregunta sobre si la empresa en la que trabaja el individuo participó en la formación realizada por él, información que en la actualidad no está disponible para los investigadores).

4 Las otras posibles respuestas varían entre 1992-1998 y 1999-2004. Antes de 1999 las otras respuestas son: «en un centro específico (a distancia u otro tipo)» y «otro caso». Desde 1999 las otras respuestas son: «asistiendo a un aula», «a distancia o por correspondencia», «por su cuenta, autoaprendizaje», $\mathrm{y}$ «otra forma». 
- si en los últimos doce meses han participado o no en algún curso de formación organizado por la empresa (V327) (esta pregunta existe desde 2002 a la vez que desparece la pregunta anterior).

En el primer caso las respuestas posibles son «nunca», «casi nunca», «algunas veces», «frecuentemente» $\mathbf{0}$ «siempre». En el segundo caso, las alternativas son «sí» $\mathbf{0}$ «no».

El cuestionario no contiene preguntas adicionales que permitan distinguir entre formación continua «formal»o «informal». Lo que puede suponerse es que las preguntas anteriores parecen referirse a actividades formativas formales. Sin embargo, hay otros aspectos de éstas que son menos claros: ¿son dichas actividades financiadas por la empresa o por el trabajador?, ¿se ofrecen en la propia empresa o fuera de la misma?, ¿se ofrecen por la empresa o son contratadas a empresas formativas externas? En cualquier caso, la información contenida en la ECVT permite conocer si un individuo ha participado en la formación que organiza su empresa. La variable que se ha construido para medir la incidencia de la formación en la empresa toma el valor uno si la empresa ofrece formación y el individuo ha participado en ella (considerando para el periodo 1999-2001 que ha participado si contesta «algunas veces», «frecuentemente» $\mathrm{O}$ «siempre» ${ }^{5}$ ) y cero en cualquier otro caso ${ }^{6}$.

En cuanto al PHOGUE, se trata de una encuesta de panel aplicada a una muestra representativa de hogares de distintos países de la Unión Europea (en España se realizó a 8.000 hogares y 17.000 individuos, aproximadamente, en su primera ola). En la actualidad, los investigadores pueden disponer de las ocho olas del panel (desde 1994 hasta 2001). Dos características fundamentales del PHOGUE son su carácter comparativo en el ámbito europeo y su diseño de panel, lo que permite entrevistar a los mismos hogares e individuos a lo largo de varios años, incluso a aquellos que cambien de domicilio siempre que esto suceda dentro de la Unión Europea, con lo que se supone que se detecta la formación de nuevos hogares y los movimientos migratorios internos.

El PHOGUE dispone de una serie de preguntas detalladas sobre características socioeconómicas de los miembros del hogar: sexo, edad, nivel de estudios, relación con la persona de referencia, situación de actividad, composición y tipo de hogar, etc. Para nuestros propósitos, también ofrece información sobre la formación. En particular, las preguntas a partir de las cuales se obtiene esta información son la (PT001) y la (PT002), que se refieren a si el individuo ha realizado formación desde enero del año anterior al de la entrevista y al tipo de cursos, diferenciando en las respuestas entre formación profesional, formación académica y una combinación de ambas. A aquellos que contestan que han realizado formación profesional se les pregunta «iQué

\footnotetext{
5 La diferencia entre incluir o no como participantes en la formación a los que contestan «casi nunca» no es relevante.

${ }^{6}$ En realidad pueden construirse dos variables relacionadas con la formación: una que indica si la empresa en la que trabaja el individuo forma o no forma a sus trabajadores (se construye a partir de la primera pregunta anterior: «sí»/»no»); y otra que indica si el individuo participa en las actividades formativas ofrecidas por la empresa. Ambas se utilizaron en el trabajo de Albert et al. (2005) para examinar la relación entre temporalidad y formación.
} 
tipo de curso de formación profesional?» (PT012) y «¿El curso fue financiado u organizado por la empresa?» (PT017).

La variable que se ha construido para conocer si un individuo ha participado en la formación de la empresa toma el valor uno si la información suministrada por los trabajadores en las preguntas anteriores nos dice que el individuo ha realizado cursos desde enero del año anterior al de la entrevista, que estos cursos son de formación profesional y que los ha financiado u organizado la empresa en la que está ocupado; en caso contrario, dicha variable toma el valor cero.

¿Cuál es la información sobre la incidencia de la formación en la empresa que ofrecen las tres encuestas que se han presentado? Para medirla, se han utilizado las variables mencionadas más arriba y construidas con cada una de las encuestas, definiendo una muestra de individuos similar (en la medida de lo posible): trabajadores asalariados con edades comprendidas entre los 16 y los 64 años (en el caso del PHOGUE, existe un criterio adicional: los trabajadores deben haber trabajado al menos 15 horas a la semana). El periodo temporal para el que se ha calculado la incidencia difiere en cada encuesta: para el PHOGUE es 1994-2001, es decir, se han considerado todas las olas del panel; para la ECVT es 1999-2004, es decir, se han tomado las ediciones de la encuesta disponibles hasta el momento; y en el caso de la EPA, se ha elegido un periodo relativamente amplio (1994-2004) para abarcar todos los años a los que se refieren las otras dos fuentes de datos. El cuadro 1 presenta la incidencia de la formación en la empresa medida con respecto al total de trabajadores asalariados en cada una de las encuestas.

\section{CUADRO 1}

TASA DE INCIDENCIA DE LA FORMACIÓN EN LA EMPRESA EN LAS DISTINTAS FUENTES DE INFORMACIÓN.

\begin{tabular}{|l|c|c|c|}
\hline & $\begin{array}{c}\text { EPA } \\
(\mathbf{1 9 9 4 - 2 0 0 4 )}\end{array}$ & $\begin{array}{c}\text { ECVT } \\
(\mathbf{1 9 9 9 - 2 0 0 4})\end{array}$ & $\begin{array}{c}\text { PHOGUE } \\
(\mathbf{1 9 9 4 - 2 0 0 1}) *)\end{array}$ \\
\hline Total & 0,3 & 27,3 & 10,2 \\
Año & & & \\
1994 & 0,2 & & 4,7 \\
1995 & 0,3 & & 6,1 \\
1996 & 0,3 & & 7,9 \\
1997 & 0,3 & & 12,5 \\
1998 & 0,4 & 28,4 & 10,9 \\
1999 & 0,6 & 26,9 & 9,6 \\
2000 & 0,3 & 32,9 & 10,6 \\
2001 & 0,3 & 26,5 & 12,6 \\
2002 & 0,3 & 25,1 & \\
2003 & 0,4 & 23,5 & \\
2004 & 0,2 & 21.862 & 26.290 \\
Número de observaciones & 539.997 & & \\
\hline
\end{tabular}


Los resultados indican que el 0,3 por ciento, el 27,3 por ciento y el 10,2 por ciento, según la EPA, la ECVT y el PHOGUE, respectivamente, de los trabajadores asalariados realizan actividades formativas en la empresa. Estas cifras sugieren (como era de esperar) la existencia de diferencias importantes en el nivel medido de formación en la empresa. Este resultado puede deberse a diversas razones.

En primer lugar, los periodos que utilizan las tres fuentes de datos para medir la formación que reciben los trabajadores son diferentes. Mientras en la EPA el periodo de referencia para medir las actividades formativas son las cuatro semanas anteriores a la entrevista, en el caso de la ECVT el trabajador ha podido realizar la formación un año antes como máximo y en el PHOGUE este periodo puede abarcar hasta casi dos años antes. Por tanto, en el caso de la EPA puede argumentarse que cuatro semanas es un periodo de tiempo demasiado corto para captar adecuadamente las actividades formativas, sobre todo si éstas son predominantemente de corta duración.

En segundo lugar, también debe tenerse en cuenta la forma en que se obtiene la información de los individuos: la EPA es una encuesta dirigida a hogares y sólo hay una persona (el informante) que suministra la información sobre todos los miembros del hogar. Este hecho es una fuente potencial de infraestimación de las actividades formativas de las personas ocupadas, si éstas no son los informantes del hogar. Algo similar sucede en el caso del PHOGUE pero no en la ECVT ya que es la persona ocupada la que proporciona información sobre sus actividades.

En tercer lugar, no hay que olvidar que se están manejado tres bases de datos distintas, cuya metodología es diferente, y que a pesar de los esfuerzos por diseñar muestras similares para medir la incidencia de la formación en la empresa, dichas muestras difieren entre las encuestas utilizadas, especialmente en el caso del PHOGUE (se restringe a los asalariados que al menos han trabajado 15 horas a la semana).

En resumen, las diferencias existentes entre las encuestas (en cuanto a su metodología, la forma de obtener la información, el periodo de referencia, etc.) podrían explicar las cifras tan distintas que se obtienen en relación con la incidencia de la formación de la empresa, aunque las diferencias en dichas cifras son realmente muy importantes.

\section{La participación de los trabajadores en la formación}

Un aspecto de la formación en la empresa que también puede investigarse con las tres fuentes de datos anteriores y al que merece la pena dedicar un cierto esfuerzo es el de la influencia de las características de los individuos, de los puestos de trabajo y de las empresas en que trabajan en la probabilidad de que los trabajadores realicen formación en la empresa. Para ello, se van a realizar dos tipos de análisis: uno meramente descriptivo, que calcula la incidencia de la formación según diversas características; y otro econométrico, que tiene en cuenta la interrelación entre las características de los individuos, de los puestos de trabajo y de las empresas.

Comenzamos con el análisis descriptivo. El cuadro 2 ofrece la incidencia de la formación en la empresa (calculada como se hizo en la sección anterior) según diversas características. 


\section{CUADRO 2}

TASA DE INCIDENCIA DE LA FORMACIÓN EN LA EMPRESA EN LAS DISTINTAS BASES DE DATOS SEGÚN CARACTERÍSTICAS

\begin{tabular}{|c|c|c|c|}
\hline & $\begin{array}{c}\text { EPA } \\
(1994-2004)\end{array}$ & $\begin{array}{c}\text { ECVT } \\
(1999-2004)\end{array}$ & $\begin{array}{c}\text { PHOGUE } \\
(1995-2001)(\mathbf{a})\end{array}$ \\
\hline Total & 0,3 & 27,3 & 10,2 \\
\hline Sexo & & & \\
\hline Varones & 0,3 & 27,1 & 9,6 \\
\hline Mujeres & 0,4 & 27,5 & 11,1 \\
\hline \multicolumn{4}{|l|}{ Edad } \\
\hline $16-24$ & 0,6 & 18,0 & 3,2 \\
\hline $25-29$ & 0,4 & 25,2 & 8,4 \\
\hline $30-44$ & 0,3 & 29,4 & 12,1 \\
\hline $45-54$ & 0,2 & 32,1 & 12,2 \\
\hline $55-64$ & 0,1 & 23,2 & 6,9 \\
\hline \multicolumn{4}{|l|}{ Estudios } \\
\hline Obligatorios & 0,2 & 14,7 & 4,0 \\
\hline Secundarios & 0,4 & 30,5 & 11,9 \\
\hline Universitarios & 0,6 & 46,5 & 18,9 \\
\hline \multicolumn{4}{|l|}{ Tipo de contrato } \\
\hline Indefinido & 0,3 & 31,9 & 13,1 \\
\hline Temporal & 0,4 & 15,1 & 4,7 \\
\hline \multicolumn{4}{|l|}{ Jornada laboral } \\
\hline Completa & 0,5 & 28,5 & 4,2 \\
\hline Parcial & 0,3 & 16,2 & 10,6 \\
\hline \multicolumn{4}{|l|}{ Antigüedad en el empleo (años) } \\
\hline$<1$ & 0,4 & 11,4 & 4,2 \\
\hline $1-3$ & 0,4 & 21,8 & 7,2 \\
\hline 4-6 & 0,3 & 25,4 & 9,4 \\
\hline $7-10$ & 0,3 & 30,7 & 12,7 \\
\hline $11-20$ & 0,3 & 36,6 & 18,0 \\
\hline $20+$ & 0,2 & 37,8 & 14,8 \\
\hline \multicolumn{4}{|l|}{ Grupo de ocupación } \\
\hline No manual-muy cualificada & 0,6 & 46,6 & 20,8 \\
\hline No manual-poco cualificada & 0,3 & 24,9 & 10,0 \\
\hline Manual-muy cualificada & 0,3 & 16,2 & 5,1 \\
\hline Manual-poco cualificada & 0,1 & 15,9 & 3,4 \\
\hline \multicolumn{4}{|l|}{ Sector institucional } \\
\hline Público & 0,7 & 49,3 & 20,9 \\
\hline Privado & 0,2 & 21,7 & 7,8 \\
\hline \multicolumn{4}{|l|}{ Tamaño de la empresa (b) } \\
\hline Categoría 1 & 0,2 & 10,4 & 4,3 \\
\hline Categoría 2 & 0,3 & 19,2 & 6,5 \\
\hline Categoría 3 & 0,4 & 26,7 & 9,6 \\
\hline
\end{tabular}


CUADRO 2 (continuación)

TASA DE INCIDENCIA DE LA FORMACIÓN EN LA EMPRESA EN LAS DISTINTAS BASES DE DATOS SEGÚN CARACTERÍSTICAS

\begin{tabular}{|l|c|c|c|}
\hline & $\begin{array}{c}\text { EPA } \\
(\mathbf{1 9 9 4 - 2 0 0 4 )}\end{array}$ & $\begin{array}{c}\text { ECVT } \\
\mathbf{( 1 9 9 9 - 2 0 0 4 )}\end{array}$ & $\begin{array}{c}\text { PHOGUE } \\
\mathbf{( 1 9 9 5 - 2 0 0 1 ) ( a )}\end{array}$ \\
\hline Categoría 4 & 0,5 & 30,3 & 12,8 \\
Categoría 5 & 0,2 & 36,7 & 14,5 \\
Categoría 6 & - & 44,5 & 18,8 \\
Categoría 7 & - & 47,7 & - \\
Categoría 8 & - & 53,9 & - \\
Rama de actividad & & & \\
Agricultura & 0,1 & 8,1 & 2,6 \\
Construcción & 0,1 & 12,8 & 2,5 \\
Energía, agua y extractivas & 0,3 & 37,7 & 9,8 \\
Transformación de metales y química & 0,2 & 28,8 & 9,9 \\
Fabricación de equipo & 0,5 & 36,8 & 7,3 \\
Otras industrias manufactureras & 0,2 & 18,9 & 6,6 \\
Servicios tradicionales & 0,2 & 19,6 & 30,7 \\
Servicios productivos & 0,4 & 37,2 & 7,4 \\
Servicios sociales & 0,7 & 45,3 & 9,8 \\
Servicios personales & 0,2 & 13,4 & 20,1 \\
Servicios públicos & 0,5 & 44,8 & 26.290 \\
Número de observaciones & 539.997 & 21.862 & \\
\hline
\end{tabular}

Notas:

(a) Dado que en 1994 no se conoce el tipo de contrato el pool se ha realizado para el periodo 1995-2001, aunque en el cuadro se aporta la incidencia de la formación para el primer año de realización de la encuesta.

(b) Las categorías de tamaño de empresa según el número de trabajadores no son comparables entre las bases de datos. En el caso de la EPA, las categorías son las siguientes: categoría 1 (menos de 10), categoría 2 (10-19), categoría 3 (2049), categoría 4 (50 o más) y categoría 5 (no sabe). En la ECVT, las categorías son las siguientes: categoría 1 (menos de 10), categoría 2 (10-25), categoría 3 (20-49), categoría 4 (50-99), categoría 5 (100-249), categoría 6 (250-499), categoría 7 (500-999) y categoría 8 (1.000 o más). En el caso del PHOGUE, las categorías son las siguientes: categoría 1 (1-4), categoría 2 (5-19), categoría 3 (20-49), categoría 4 (50-99), categoría 5 (100-499) y categoría 6 (500 o más).

En cuanto a las características de los trabajadores, las tres bases de datos confirman que la incidencia de la formación es mayor entre las mujeres que entre los varones y que crece claramente con el nivel de estudios (quienes tienen estudios universitarios presentan una incidencia que es unas 3-4 veces superior a la de quienes tienen estudios obligatorios o inferiores). Sin embargo, los resultados referidos a la edad son muy distintos: en el caso de la ECVT y el PHOGUE, la relación entre edad e incidencia de la formación tiene forma de U invertida (la incidencia crece con la edad hasta los 50 años y luego disminuye), mientras que en la EPA dicha relación tiene pendiente negativa (la incidencia es más elevada entre los más jóvenes y se va reduciendo con la edad). 
Por lo que respecta a las características de los puestos de trabajo, hay un resultado que se da con las tres encuestas utilizadas: la incidencia de la formación es más elevada entre las ocupaciones de naturaleza no manual frente a las de naturaleza manual y entre las ocupaciones muy cualificadas frente a las no cualificadas. Sin embargo, en el resto de características de los puestos de trabajo hay una oposición clara entre la ECVT y el PHOGUE, por un lado, y la EPA, por otro lado. Así, en las dos primeras encuestas se observa que la incidencia de la formación en la empresa crece con la antigüedad en el empleo y es mayor entre los trabajadores con contrato indefinido que entre los trabajadores con contrato temporal, mientras que en la última sucede lo contrario.

Finalmente, es en el grupo de las características de la empresa donde trabaja el individuo en el que aparece un mayor acuerdo entre los resultados de las tres bases de datos. Así, la incidencia de la formación crece con el tamaño de la empresa (aunque esta variable se agrupa de forma distinta en cada encuesta) y es mayor en las empresas pertenecientes al sector público que entre las pertenecientes al sector privado. De hecho, incluso las ramas de actividad en que la incidencia de la formación es más elevada son casi las mismas en las tres bases de datos (servicios productivos y servicios públicos, a los que se añaden fabricación de equipo y servicios sociales en la EPA y la ECVT).

Antes de pasar al análisis econométrico, un comentario que debe hacerse sobre los datos y los resultados obtenidos. Los periodos a los que se refieren las actividades formativas pueden diferir del periodo (o del momento) en que se obtiene el resto de la información suministrada por los entrevistados en las encuestas. Así, en las tres encuestas el tipo de contrato y otras características del puesto de trabajo y de la empresa en la que se encuentra ocupado el trabajador se refieren al momento de contestar el cuestionario, mientras que, como se ha comentado más arriba, la formación realizada se refiere a un periodo temporal anterior más amplio (sobre todo en el caso de la ECVT y del PHOGUE). Es posible, por tanto, que en el momento en el que el individuo ha realizado la formación que ha organizado la empresa tuviese un contrato distinto al actual, estuviese en una ocupación diferente, etc. Este hecho puede influir de algún modo en los resultados presentados más arriba.

Para poder profundizar en el análisis de la participación en la formación de las empresas hay que tener en cuenta que las características personales de los trabajadores y las características de los puestos de trabajo y de las empresas en que trabajan se encuentran interrelacionadas. Por ello, resulta necesario estimar un modelo multivariante. En particular, se ha estimado un modelo de elección discreta que explica la probabilidad de que un trabajador participe en la formación que organiza su empresa, utilizando para ello cada una de las tres encuestas. De este modo, la variable dependiente toma el valor uno si el trabajador participa en las actividades formativas de la empresa y el valor cero en caso contrario, siendo la variable dependiente cada una de las variables dicotómicas empleada previamente en cada una de las encuestas ${ }^{7}$.

7 Es evidente que para que un trabajador participe en las actividades formativas de la empresa es un requisito previo que éstas deben existir, es decir, si la empresa no realiza formación sus empleados 
En la estimación, se ha controlado por diversas características de los trabajadores, de la empresa y de los puestos. Los regresores hacen referencia, por una parte, al sexo, la edad y el nivel educativo del individuo; por otra parte, al tipo de contrato, la jornada laboral, la antigüedad en el empleo y la ocupación a la que pertenece el puesto de trabajo; y finalmente, al sector institucional (público o privado), la rama de actividad económica y el tamaño de la empresa. También se han incluido como variables de control el año de realización de la encuesta y la comunidad autónoma de residencia. El cuadro 3 presenta los resultados de la estimación. Para facilitar la lectura de los resultados, en el cuadro se ofrecen los efectos marginales de cada categoría con respecto a las del individuo de referencia. Los coeficientes correspondientes se muestran en el cuadro A.1 del apéndice.

Comenzando por las variables que captan las características individuales, los resultados indican que, una vez que se controla por el resto de las características observables, el efecto de algunas variables es diferente al contemplado en el análisis descriptivo mientras que el de otras se mantiene. Así, en contra de lo obtenido previamente, las mujeres presentan una menor probabilidad de participar en actividades de formación en la empresa en comparación con los varones (un 4 por ciento menos, excepto en el caso del PHOGUE, con el que esta variable no es significativa). Algo similar sucede con la edad: se confirma la relación negativa entre edad e incidencia encontrada previamente en la EPA y la relación en forma de U invertida en el caso del PHOGUE, pero esta última relación desaparece en la ECVT. En cualquier caso, con las tres fuentes de datos se obtiene que son los más mayores (55-64 años) quienes presentan la probabilidad más baja de recibir formación en la empresa: en comparación con los trabajadores de 16-24 años, su probabilidad es un 9 por ciento menor con el PHOGUE y un 18 por ciento menor con la EPA.

En el caso de los estudios, también se confirma el resultado anterior de que a mayor nivel de estudios mayor probabilidad de participar en las actividades formativas de las empresas. Esto vendría a indicar que los trabajadores más cualificados se incorporan a puestos en ocupaciones y sectores que requieren elevadas cualificaciones $\mathrm{y}$, por tanto, como tienen en principio más capacidad para aprender, presentan una mayor probabilidad de recibir formación que otros trabajadores menos cualificados en ocupaciones con menores requerimientos educativos. Además, como los programas de formación son costosos, las empresas querrán garantizar el rendimiento de sus inversiones, por lo que elegirán trabajadores con niveles de educación altos. Todo ello puede generar un círculo virtuoso en las inversiones en capital humano (Lynch y Black, 1998; Peraita, 2005).

Con respecto a las características de los puestos de trabajo, resulta muy relevante el tipo de tareas que realiza el trabajador. De este modo, la probabilidad de recibir

no pueden recibirla. No tener en cuenta este hecho puede generar un sesgo en el efecto de determinadas características sobre la probabilidad de recibir formación. Trabajos que analizan esta cuestión son Oosterbeek (1996) para Holanda, y Albert et al. (2005) y Abellán y Felgueroso (2005) para España (con la ECVT). 
CUADRO 3

MODELO LOGÍSTICO DE LA PROBABILIDAD DE QUE UN TRABAJADOR PARTICIPE EN LA FORMACIÓN DE LA EMPRESA

\begin{tabular}{|c|c|c|c|c|c|c|}
\hline & \multicolumn{2}{|c|}{ EPA (1994-2004) } & \multicolumn{2}{|c|}{ ECVT (1999-2004) } & \multicolumn{2}{|c|}{ PHOGUE (1995-2001)(a) } \\
\hline & Efecto Marg. & Sig. & Efecto Marg. & Sig. & Efecto Marg. & Sig. \\
\hline Sexo (Varón) & & & & & & \\
\hline $\begin{array}{l}\text { Mujer } \\
\text { Edad (16-24) }\end{array}$ & $-0,045$ & **** & $-0,044$ & $* * *$ & $-0,024$ & \\
\hline $25-29$ & $-0,157$ & $* * *$ & $-0,022$ & & 0,044 & \\
\hline $30-44$ & $-0,211$ & *** & $-0,031$ & & 0,068 & ** \\
\hline $45-54$ & $-0,222$ & $* * *$ & $-0,033$ & & 0,043 & \\
\hline $55-64$ & $-0,180$ & *** & $-0,140$ & $* * *$ & $-0,090$ & $* *$ \\
\hline Estudios (Obligatorios) & & & & & & \\
\hline Secundarios & 0,096 & $* * *$ & 0,125 & $* * *$ & 0,138 & $* * *$ \\
\hline Universitarios & 0,114 & $* * *$ & 0,164 & $* * *$ & 0,174 & $* * *$ \\
\hline Tipo de contrato (Indefinido) & & & & & & \\
\hline $\begin{array}{l}\text { Temporal } \\
\text { Jornada laboral (Parcial) }\end{array}$ & 0,109 & $* * *$ & $-0,081$ & $* * *$ & $-0,072$ & **** \\
\hline Completa & 0,082 & $* * *$ & 0,071 & $* * *$ & $-0,073$ & ** \\
\hline $\begin{array}{l}\text { Antigüedad en el empleo (<1 año) } \\
1-3\end{array}$ & & & & & & \\
\hline $1-3$ & 0,069 & $* * *$ & 0,127 & $* * *$ & 0,078 & $* * *$ \\
\hline $4-6$ & 0,050 & & 0,143 & $* * *$ & 0,112 & $* * *$ \\
\hline $7-10$ & 0,052 & & 0,168 & $* * *$ & 0,127 & $* * *$ \\
\hline $11-20$ & 0,060 & ** & 0,168 & $* * *$ & 0,166 & $* * *$ \\
\hline $20+$ & 0,031 & & 0,177 & $* * *$ & 0,151 & $* * *$ \\
\hline Ocupación (No manual-muy cualific.) & & & & & & *** \\
\hline No manual-poco cualificada & $-0,087$ & $* * *$ & $-0,081$ & $* * *$ & $-0,048$ & $* * *$ \\
\hline Manual-muy cualificada & $-0,052$ & ** & $-0,142$ & $* * *$ & $-0,145$ & $* * *$ \\
\hline Manual-poco cualificada & $-0,189$ & $* * *$ & $-0,169$ & $* * *$ & $-0,181$ & $* * *$ \\
\hline Sector (Público) & & & & & & \\
\hline $\begin{array}{l}\text { Privado } \\
\text { Tamaño de la empresa(b) (Categoría 1) }\end{array}$ & $-0,177$ & $* * *$ & $-0,068$ & $* * *$ & $-0,084$ & *** \\
\hline Categoría 2 & 0,028 & & 0,131 & $* * *$ & 0,080 & $* * *$ \\
\hline Categoría 3 & 0,071 & $* * *$ & 0,190 & $* * *$ & 0,115 & $* * *$ \\
\hline Categoría 4 & 0,118 & $* * *$ & 0,203 & $* * *$ & 0,159 & $* * *$ \\
\hline Categoría 5 & $-0,049$ & $* *$ & 0,223 & $* * *$ & 0,173 & $* * *$ \\
\hline Categoría 6 & - & & 0,222 & $* * *$ & 0,164 & $* * *$ \\
\hline Categoría 7 & - & & 0,224 & $* * *$ & - & \\
\hline Categoría 8 & - & & 0,224 & $* * *$ & - & \\
\hline Rama de actividad (Agricultura) & & & & & & \\
\hline Construcción & $-0,065$ & & 0,048 & & $-0,062$ & \\
\hline Energía, agua y extractivas & 0,070 & & 0,148 & $* * *$ & 0,114 & \\
\hline Transformación de metales y química & 0,032 & & 0,128 & $* * *$ & 0,077 & \\
\hline Fabricación de equipo & 0,128 & *** & 0,157 & $* * *$ & 0,102 & \\
\hline Otras industrias manufactureras & $-0,048$ & & 0,064 & & 0,063 & \\
\hline Servicios tradicionales & $-0,001$ & & 0,102 & $* * *$ & 0,032 & \\
\hline Servicios productivos & 0,143 & *** & 0,171 & $* * *$ & 0,214 & $* * *$ \\
\hline Servicios sociales & 0,070 & & 0,131 & $* * *$ & 0,027 & \\
\hline Servicios personales & 0,007 & & 0,052 & & 0,060 & \\
\hline Servicios públicos & $-0,001$ & & 0,102 & $* * *$ & 0,086 & \\
\hline Constante & $-0,032$ & **** & $-0,156$ & $* * *$ & $-0,076$ & *** \\
\hline Número observaciones & 539.997 & & 21.862 & & 26.290 & \\
\hline
\end{tabular}

Notas: (a) y (b) Véase el cuadro 2. Las categorías de referencia de cada variable aparecen entre paréntesis. En las estimaciones, se han incluido controles de las comunidades autónomas de residencia y de los años de las encuestas. Los asteriscos indican significatividad estadística al 1 por ciento $(* * *)$ y al 5 por ciento $(* *)$, respectivamente. 
formación es mayor si el puesto lleva asociado unas tareas de naturaleza no manual (frente a tareas manuales) y con alta cualificación (frente a baja cualificación). Así, con las tres bases de datos se obtiene que los trabajadores en ocupaciones manuales no cualificadas presentan casi un 20 por ciento menos de probabilidad de participar en actividades formativas en comparación con los trabajadores en ocupaciones no manuales muy cualificadas. Esto significa que la probabilidad de recibir formación aumenta a medida que se consideran puestos de trabajo en los que el control de la empresa es menor y los costes asociados a la no realización de las tareas por parte del trabajador son mayores (Altonji y Spletzer, 1991).

Los resultados referidos al tipo de contrato y a la antigüedad en el empleo son de interés, ya que difieren según la base de datos que se utilice. En efecto, manteniendo constantes las características estadísticamente observables de las empresas, los trabajadores con contratos temporales presentan aproximadamente un 8 por ciento menos de probabilidad de participar en la formación de la empresa si se utilizan los datos de la ECVT y del PHOGUE y un 11 por ciento más si se utilizan los datos de la EPA ${ }^{8}$. Al mismo tiempo, dicha probabilidad crece con la antigüedad en el primer caso y muestra una relación poco clara en el segundo caso (quienes presentan mayor probabilidad son los trabajadores con una antigüedad entre uno y tres años).

Por lo que respecta a las variables de las empresas en que trabajan los individuos, resulta interesante destacar que, en línea con el análisis descriptivo, trabajar en ciertos sectores de actividad económica (como servicios productivos o fabricación de equipo) implica una mayor probabilidad de participar en la formación de la empresa. Lo mismo sucede si la empresa pertenece al sector público: la probabilidad de participar en actividades formativas es un 7-8 por ciento menor según la ECVT y el PHOGUE y un 18 por ciento menor según la EPA en el caso de los trabajadores del sector privado en comparación con los trabajadores del sector público. Además, dicha probabilidad crece con el tamaño de la empresa medida por el número de trabajadores. Este resultado recoge el hecho de que las empresas grandes presentan economías de escala en la provisión de formación y, además, retienen a los trabajadores formados a través de salarios más elevados y de mayores perspectivas de promoción (Black et al., 1999).

En resumen, los resultados obtenidos del análisis sobre el perfil de los trabajadores que participan en la formación de la empresa muestran que algunas características presentan el mismo efecto cualquiera que sea la base de datos empleada para llevar a cabo la investigación. Así, todas ellas coinciden en que es más probable que un trabajador participe en actividades de formación cuanto mayor sea su nivel de estudios, cuanto más cualificada sea la ocupación en la que trabaja y cuanto más grande es la empresa para la que lo hace. Sin embargo, los efectos de otras características di-

8 También existen diferencias según el tipo de contrato temporal: los trabajadores con contratos de formación, aprendizaje o prácticas presentan una probabilidad mayor de recibir formación que los trabajadores con contratos temporales de otro tipo (véase Albert et al. (2005) para un análisis más detallado de la relación entre temporalidad y formación). 
fieren según la fuente estadística utilizada: de acuerdo con la EPA, la mayor incidencia de la formación corresponde a trabajadores jóvenes, con contratos temporales y con poca antigüedad en su puesto de trabajo (entre uno y tres años), mientras que según la ECVT y el PHOGUE la mayor probabilidad se da entre los trabajadores maduros, con contratos indefinidos y con más antigüedad en el puesto de trabajo. Esto puede deberse a la distinta naturaleza o duración de las actividades formativas que se recogen en cada una de dichas fuentes. A investigar esta cuestión se dedica la sección siguiente.

\section{La intensidad de la formación}

Una vez estudiada la incidencia de la formación en la empresa y el perfil de los trabajadores que participan en ella, nos preguntamos por su intensidad medida como el tiempo durante el cual el trabajador realiza dicha formación. Como vamos a ver a continuación, cada una de las encuestas utilizadas en este trabajo mide de forma diferente la duración de la formación, por lo que la comparación entre ellas va a resultar difícil.

La EPA pregunta por la duración total de la formación (recordemos que ésta se refiere a las cuatro semanas anteriores a la entrevista) y ofrece una respuesta en intervalos, que varían a partir de 1999. Desde ese año los intervalos son: «Menos de 1 semana», «De 1 semana a menos de 1 mes», «De 1 mes a menos de 3 meses», «De 6 meses a menos de 1 año», «De 1 año a menos de 2 años», «De 2 años a menos de 4 año», «4 años o más» y «No definida». Antes de 1999 se ofrecían los cinco primeros intervalos y a continuación se daba la opción de «1 año o más», sin considerar tampoco la categoría de «No definida». Además, la EPA también realiza una pregunta sobre el número habitual de horas de formación a la semana. De esta manera la información que ofrece la EPA es bastante completa. El problema es el pequeño porcentaje de individuos que realizan formación en la empresa, que no permite tener un número de observaciones suficiente que posibilite realizar para un año estudios descriptivos sobre las características del individuo y del puesto de trabajo. Este problema se resuelve agregando los cuatro trimestres de cada año desde 1999 hasta 2004, es decir, creando un fichero con los microdatos correspondientes a todos los trimestres comprendidos entre el primero de 1999 y el cuarto de $2004^{9}$. Se ha preferido no realizar el análisis para años anteriores a 1999 con el propósito de garantizar la homogeneidad de la pregunta que hace referencia a la duración completa de la formación.

En la ECVT, desde el año 2001 en adelante, a todos aquellos que contestan que han realizado formación en la empresa les hacen la siguiente pregunta: «¿En los últimos doce meses, ¿qué número de días (en días completos) ha asistido a cursos organizados y financiados por su empresa?» (pregunta V312). De esta manera, la ECVT

9 Para corregir la ponderación se ha dividido el factor de ponderación entre cuatro. 
ofrece información sobre los días completos de formación en un año. El problema que presenta esta pregunta es que la formación en la empresa no siempre se realiza en días completos, ya que en ocasiones sólo se dedican unas horas en el día.

Finalmente, el PHOGUE dispone de dos preguntas que palian en parte las limitaciones de la pregunta anterior de la ECVT. En la primera (PT018), se le pregunta al trabajador si realizó la formación a tiempo completo, a tiempo parcial o por correspondencia. En la segunda (PT019), a aquellos que contestan que las actividades formativas fueron a tiempo parcial se les pregunta por el número de horas a la semana que dedicaron.

El cuadro 4 ofrece las medidas de la intensidad de la formación en la empresa aproximada por su duración calculadas con los datos correspondientes a las tres fuentes estadísticas comentadas.

\section{CUADRO 4}

MEDIDAS DE LA INTENSIDAD DE LA FORMACIÓN EN LA EMPRESA (TRABAJADORES QUE REALIZAN FORMACIÓN)

\begin{tabular}{|c|c|c|c|c|c|}
\hline & $\%$ & Media & $\begin{array}{l}\text { Me- } \\
\text { diana }\end{array}$ & Moda & $\begin{array}{l}\text { Desv. } \\
\text { típica }\end{array}$ \\
\hline $\begin{array}{l}\text { EPA (1999-2004) (3.654 observaciones) } \\
\text { Duración total } \\
\text { Menos de } 1 \text { semana } \\
\text { De } 1 \text { semana a < de } 1 \text { mes } \\
\text { De } 1 \text { a }<\text { de } 3 \text { meses } \\
\text { De } 3 \text { a }<\text { de } 6 \text { meses } \\
\text { De } 6 \text { a }<\text { de } 1 \text { año } \\
\text { De } 1 \text { año a }<\text { de } 2 \text { años } \\
\text { De } 2 \text { a }<\text { de } 4 \text { años } \\
4 \text { años o más } \\
\text { No definida } \\
\text { No habitual de horas de formación a la semana } \\
\text { ECVT (2001-2004) ( } 3.879 \text { observaciones) } \\
\text { No de días completos que dedicó a la formación } \\
\text { (últimos } 12 \text { meses) } \\
\text { PHOGUE (1995-2001) (2.675 observaciones) } \\
\text { ¿Cómo fue la formación realizada (desde enero } \\
\text { del último año)? } \\
\text { A tiempo completo } \\
\text { A tiempo parcial } \\
\text { Por correspondencia } \\
\text { No habitual de horas de formación a la semana } \\
\text { para aquellos que realizaron la formación a } \\
\text { tiempo parcial }\end{array}$ & $\begin{array}{r}9,5 \\
13,0 \\
7,5 \\
7,1 \\
14,4 \\
13,9 \\
14,9 \\
7,0 \\
12,8\end{array}$ & 15,0 & 15 & 20 & $\begin{array}{l}12,2 \\
25,0\end{array}$ \\
\hline
\end{tabular}


Comenzado por la información ofrecida por el PHOGUE, cabe destacar que un 63,9 por ciento de los encuestados recibieron la formación sólo a tiempo parcial. Además, el número de horas de formación a la semana supone más de una cuarta parte de la jornada laboral normal, con 10 horas de formación de media. El problema de esta estas cifras es que no se sabe cuál fue la duración de las actividades formativas.

La información proporcionada por los datos de la EPA permite conocer, por una parte, el número habitual de horas de formación a la semana recibidas por el conjunto de los trabajadores que recibieron formación, a tiempo completo o parcial. Dado que, como muestran los datos del PHOGUE, una parte mayoritaria de los trabajadores dice recibir formación a tiempo parcial, el resultado con la EPA debería ser que al considerar el conjunto de trabajadores que recibieron formación el número medio de horas dedicado a la misma fuese más elevado. En efecto, este es el resultado que se obtiene, ya que los trabajadores que realizaron formación dedicaron en media casi 18 horas a la semana, según la EPA. Por otra parte, la información de esta encuesta también permite conocer la distribución de la duración total de la formación (algo que no ofrece el PHOGUE), al separar el colectivo de los trabajadores que han recibido formación en dos grandes grupos: aquellos que han recibido formación por un periodo inferior al año - el 51,5 por ciento - y aquellos que han recibido formación durante un año o más - el 36 por ciento - (el resto no especifica la duración). Esto significa que más de un tercio de los trabajadores que realizan actividades formativas en las empresas las llevan a cabo de forma continuada a lo largo del tiempo, siendo aquéllas de larga duración.

En cuanto a los datos de la ECVT, éstos ofrecen el número de días completos durante los cuáles los trabajadores han recibido formación en el periodo de un año. Esta información es quizás la más difícil de analizar debido a la gran dispersión que reflejan los resultados: en media los trabajadores declaran haber recibido 15 días completos de formación pero la respuesta más frecuente (moda) es de sólo 5 días de formación. Además, dicha media implicaría un número de horas a la semana cercano a 3, lo cual es realmente bajo en comparación con las cifras proporcionadas por la EPA y el PHOGUE.

Para finalizar el análisis de la intensidad de la formación en la empresa, el cuadro 5 presenta la información sobre dicha intensidad, aproximada por su duración, según diversas características de los trabajadores, de los puestos de trabajo y de las empresas.

Dado que, como ya se ha comentado previamente, cada una de las tres encuestas utilizadas mide de forma diferente la duración de la formación, no es de extrañar que la influencia de determinadas características varíe según la fuente de datos analizada y que parezca aconsejable interpretar estas diferencias con cierta cautela.

Esto es lo que sucede en el caso del sexo: la información obtenida con la EPA muestra que la intensidad de la formación es prácticamente igual entre varones y mujeres, mientras que con la ECVT las mujeres exhiben una intensidad algo mayor y con el PHOGUE (cuyos datos se refieren a la formación a tiempo parcial) se obtiene el resultado contrario, es decir, son los varones los que presentan una intensidad mayor de la formación recibida.

En cuanto a las diferencias en la intensidad por tipo de contrato, los resultados obtenidos a partir de las tres encuestas coinciden en señalar que existe una diferencia po- 
CUADRO 5

MEDIDAS DE LA INTENSIDAD DE LA FORMACIÓN EN LA EMPRESA SEGÚN ALGUNAS CARACTERÍSTICAS DE LOS TRABAJADORES, DEL PUESTO DE TRABAJO Y DE LA EMPRESA

\begin{tabular}{|c|c|c|c|c|c|c|c|c|}
\hline & \multicolumn{2}{|c|}{$\begin{array}{c}\text { EPA }(1999-2004) \\
\text { No habitual de horas }^{0} \\
\quad \text { a la semana }\end{array}$} & \multicolumn{2}{|c|}{$\begin{array}{l}\text { ECVT (2001-2004) } \\
\text { No días completos }^{0} \\
\text { en doce meses }\end{array}$} & \multicolumn{2}{|c|}{$\begin{array}{l}\text { PHOGUE (1995-2001) } \\
\mathrm{N}^{0} \text { habitual de horas } \\
\text { a la semana (formación } \\
\text { a tiempo parcial) }\end{array}$} \\
\hline & & & Media & D. típica & Media & D. típica & Media & D. típica \\
\hline \multicolumn{3}{|l|}{ Sexo } & & & & & & \\
\hline \multicolumn{3}{|l|}{ Varones } & 17,7 & 12,2 & 14,2 & 23,0 & 10,9 & 6,9 \\
\hline \multirow{2}{*}{\multicolumn{3}{|c|}{$\begin{array}{l}\text { Mujeres } \\
\text { Tipo de contrato }\end{array}$}} & 17,9 & 12,3 & 16,5 & 28,0 & 9,1 & 7,0 \\
\hline \multirow{2}{*}{\multicolumn{3}{|c|}{$\begin{array}{l}\text { Tipo de contrato } \\
\text { Fijo }\end{array}$}} & & & & & & \\
\hline & & & 13,7 & 11,3 & 14,9 & 24,1 & 10,2 & 7,1 \\
\hline \multirow{2}{*}{\multicolumn{3}{|c|}{$\begin{array}{l}\text { Temporal } \\
\text { Antigüedad en el empleo }\end{array}$}} & 20,9 & 12,0 & 16,2 & 29,6 & 10,6 & 6,7 \\
\hline & & & 201 & 116 & 162 & 289 & 116 & 78 \\
\hline \multicolumn{3}{|l|}{$1-3$} & $\begin{array}{l}20,1 \\
18,9\end{array}$ & $\begin{array}{l}11,0 \\
13,2\end{array}$ & $\begin{array}{l}10,2 \\
15,8\end{array}$ & $\begin{array}{l}28,9 \\
27,0\end{array}$ & $\begin{array}{l}11,0 \\
10,3\end{array}$ & $\begin{array}{l}7,8 \\
6,6\end{array}$ \\
\hline \multicolumn{3}{|l|}{$4-6$} & 16,3 & 13,2 & 16,6 & 28,2 & 9,6 & 6,6 \\
\hline \multicolumn{3}{|l|}{$7-10$} & 12,6 & 10,7 & 16,1 & 29,6 & 10,2 & 7,8 \\
\hline \multicolumn{3}{|l|}{$11-20$} & 12,5 & 10,7 & 13,9 & 20,6 & 9,5 & 6,6 \\
\hline \multicolumn{3}{|c|}{$\begin{array}{l}20+ \\
\text { Tamaño de la empresa }\end{array}$} & 12,0 & 10,4 & 13,9 & 21,7 & 10,5 & 7,1 \\
\hline EPA & ECVT & PHOGUE & & & & & & \\
\hline$<10$ & $<10$ & $1-4$ & 16,3 & 12,2 & 15,8 & 26,7 & 10,5 & 7,5 \\
\hline $10-19$ & $10-25$ & $5-19$ & 16,6 & 11,4 & 16,1 & 31,4 & 10,2 & 7,5 \\
\hline 20-49 & $26-49$ & $20-49$ & 14,0 & 11,0 & 15,6 & 22,5 & 8,7 & 6,5 \\
\hline 50 ó más & $50-99$ & 50-99 & 18,1 & 12,7 & 15,6 & 26,2 & 9,6 & 6,8 \\
\hline \multirow[t]{4}{*}{ No sabe } & $100-249$ & $100-499$ & 20,1 & 11,5 & 13,6 & 23,7 & 10,6 & 6,5 \\
\hline & $250-499$ & $>=500$ & - & - & 16,4 & 26,0 & 11,2 & 7,2 \\
\hline & $500-999$ & & - & - & 14,1 & 19,1 & - & - \\
\hline & $>=1000$ & & - & - & 14,5 & 23,1 & - & - \\
\hline \multicolumn{9}{|c|}{ Grupo de ocupación } \\
\hline \multirow{4}{*}{\multicolumn{3}{|c|}{$\begin{array}{l}\text { No manual-cualificada } \\
\text { No manual-poco cualificada } \\
\text { Manual-cualificada }\end{array}$}} & 18,0 & 13,0 & 16,8 & 26,3 & 9,8 & 7,3 \\
\hline & & & 17,2 & 11,7 & 13,3 & 21,5 & 10,8 & 6,5 \\
\hline & & & 18,0 & 12,0 & 12,9 & 25,0 & 10,1 & 6,6 \\
\hline & & & 17,4 & 10,6 & 14,3 & 25,9 & 11,2 & 7,3 \\
\hline
\end{tabular}


sitiva (sobre todo en el caso de la EPA) a favor de aquellos que poseen un contrato temporal. Es decir, los trabajadores con contrato temporal (en el periodo de referencia de la encuesta) tienen menos probabilidad de recibir formación según la ECVT y el PHOGUE pero más según la EPA (tal como vimos en las secciones anteriores), pero una vez que la reciben las actividades formativas son algo más intensas según la ECVT y el PHOGUE y claramente más intensas según la EPA.

Con respecto a la intensidad de la formación según la antigüedad de los trabajadores, de nuevo la información obtenida a partir de las tres fuentes de datos coincide en señalar a aquellos con una antigüedad menor (menos de un año) como los que llevan a cabo una formación más intensa. Sin embargo, más allá de esta coincidencia la relación entre antigüedad y formación es diferente según la base de datos utilizada. Así, la EPA muestra una relación claramente negativa, aunque a partir de cierta antigüedad (más de seis años) la intensidad de la formación se mantiene constante. Algo similar sucede con el PHOGUE, si bien la reducción inicial de la intensidad con la antigüedad es mucho más limitada. Finalmente, le ECVT sugiere que la intensidad de la formación es similar para todos aquellos con una antigüedad inferior a diez años, siendo más baja en el caso de quienes superan dicha antigüedad.

Especialmente difícil resulta el poder establecer una relación entre el tamaño de las empresas y la intensidad de la formación de los trabajadores, ya que la forma en que ha podido ser codificada la información sobre el tamaño de las empresas varía en cada encuesta. En cualquier caso, parece detectarse una cierta relación en forma de U entre el tamaño y la intensidad de la formación que reciben los trabajadores según la EPA y el PHOGUE: las empresas más pequeñas y, sobre todo, las más grandes son las que exhiben intensidades más elevadas, mientras que las empresas medianas (20-49 trabajadores) presentan los valores más bajos. Sin embargo, la ECVT no ofrece este resultado sino más bien una falta de relación entre tamaño e intensidad de la formación.

Finalmente, si se considera el grupo ocupacional del puesto de trabajo, lo que se obtiene es que, según la ECVT, la intensidad de la formación en la empresa es mayor para los trabajadores que pertenecen a ocupaciones no manuales cualificadas en comparación con el resto de grupos, mientras que con la EPA y el PHOGUE las diferencias no resultan importantes (si acaso, con este último parece que son los trabajadores en ocupaciones manuales no cualificadas quienes se benefician de actividades formativas más intensas, aunque en las secciones anteriores vimos que se trata del grupo con menor probabilidad de recibir formación en la empresa).

\section{Conclusiones}

En este artículo se han utilizado tres encuestas (la Encuesta de Población Activa, la Encuesta de Calidad de Vida en el Trabajo y el Panel de Hogares de la Unión Europea) con el propósito de comparar la incidencia de la formación en la empresa, de examinar las características de los trabajadores que reciben esta formación, de medir 
la duración de las actividades formativas en la empresa y de investigar las características de los trabajadores asociadas a duraciones más largas o más cortas.

Los resultados obtenidos en cuanto a la incidencia de la formación en la empresa indican que esta medida de la formación difiere entre las tres encuestas utilizadas de una forma notable, no sólo porque el periodo de referencia de la formación es muy distinto (cuatro semanas en la EPA y casi dos años en el PHOGUE) sino debido a otras razones vinculadas al modo de obtener la información.

En cuanto al análisis sobre el perfil de los trabajadores que participan en la formación de la empresa, los resultados revelan que ciertas características presentan el mismo efecto con las tres bases de datos (es más probable que un trabajador participe en actividades de formación cuanto mayor sea su nivel de estudios, cuanto más cualificada sea la ocupación en la que trabaja y cuanto más grande es la empresa para la que lo hace). Lo relevante es que los efectos de otras características difieren de manera importante según la encuesta empleada: mientras que con la EPA la mayor incidencia de la formación corresponde a los trabajadores jóvenes, con contratos temporales y con poca antigüedad en su puesto de trabajo (entre uno y tres años), con la ECVT y el PHOGUE la mayor probabilidad se presenta entre los trabajadores maduros, con contratos indefinidos y con más antigüedad en el puesto de trabajo.

Estos resultados tan dispares pueden atribuirse a la distinta naturaleza o duración de las actividades formativas que se recogen en cada una de dichas fuentes, como parece desprenderse del análisis de la intensidad de la formación en la empresa. En este caso, las medidas que pueden construirse con cada una de las encuestas utilizadas son diferentes, lo que hace que la comparación entre ellas resulte difícil. Así, se obtienen algunos resultados similares (como que los trabajadores con contratos temporales y con menor antigüedad son los que presentan una intensidad mayor de la formación que reciben), pero otros son muy distintos.

Para finalizar, merece la pena llamar la atención sobre la proliferación en los últimos años en España de la literatura sobre participación de los trabajadores en la formación de la empresa, al tiempo que escasean los estudios sobre la intensidad de dicha formación. Aunque la información existente sobre dicha intensidad no es la mejor y está lejos de ser la deseable, los análisis exploratorios realizados en este trabajo invitan a profundizar en este aspecto de la formación en la empresa.

\section{Referencias bibliográficas}

[1] ABELLÁN, C. y F. FELGUEROSO (2005): «Formación no reglada y productividad», Presupuesto y Gasto Público, 38, 1-29.

[2] ACEMOGLU, D. y J. PISHKE (1998): «Why do firms train? Theory and evidence», Quarterly Journal of Economics, 113(1), 79-119.

[3] ACEMOGLU, D. y J. PISHKE. (1999): «Beyond Becker: training in imperfect labour markets», The Economic Journal, 109, F112-F142.

[4] ALBA, A. (1994): «Formal training, temporary contracts, productivity and wages in Spain», Oxford Bulletin of Economics and Statistics, 56(2), 151-170. 
[5] ALBA, A. y M. TUGORES (2000): «Un estudio microeconómico sobre los determinantes y efectos de la formación en España», 351-20, en F. Sáez (coord.), Formación y Empleo, Fundación Argentaria.

[6] ALBERT, C., C. GARCÍA-SERRANO y V. HERNANZ (2005): «Firm-provided training and temporary contracts», Spanish Economic Review, 7(1), 67-88.

[7] ALBERT, C., C. GARCÍA-SERRANO y V. HERNANZ (2007): «Los determinantes de la formación en la empresa y sus rendimientos», en VV.AA. El mercado de trabajo europeo en el proceso de convergencia económica y social: un análisis basado en el Panel de Hogares de la Unión Europea (PHOGUE), 1994-2001, Fundación Caixa Galicia.

[8] ALTONJI, J. y J. SPLETZER (1991): «Worker characteristics, job characteristics, and the receipt of on-the-job training», Industrial and Labor Relations Review, 45(1), 58-79.

[9] BARRON, J.; M. BERGER y D. BLACK (1997): «How well do we measure the training?», Journal of Labour Economics, 15(2), 507-28.

[10] BOOTH, A., M. FRANCESCONI y G. ZOEGA (2003): «Unions, work-related training, and wages: evidence for British men», Industrial and Labor Relations Review, 57 (1), 68-91.

[11] BECKER, G. S. (1962): «Investment in human capital», Journal of Political Economy, 70, 9-49.

[12] BLACK, D., B. NOEL y Z. WANG (1999): «On-the-job training, establishment size, and firm size: evidence for economies of scale in the production of human capital», Southern Economic Journal, 66(1), 82-100.

[13] CAPARRÓS, A., M.L. NAVARRO y M. RUEDA, M. (2004): «Efectos de la temporalidad sobre la formación recibida durante el empleo», Cuadernos de Economía, 27, 51-73.

[14] GREENHALGH, C. y M. STEWART (1987): «The effects and determinants of training», Oxford Bulletin of Economics and Statistics, 49(2), 171-190.

[15] KATZ, E. y A. ZIDERMAN (1990): «Investment in general training: the role of information and labour mobility», The Economic Journal, 100, 1147-1158.

[16] LOEWENSTEIN, M.A. y J.R. SPLETZER (1999): «General and specific training: evidence and implications», Journal of Human Resources, 34 (4), 710-733.

[17] LYNCH, L. (1991): «The role of off-the-job vs. on-the-job training for the mobility of women workers», American Economic Review, 81(2), 151-156.

[18] LYNCH, L. (1992): «Private-sector training and the earnings of young workers», American Economic Review, 82(1), 299-312.

[19] LYNCH, L. y S. BLACK (1998): «Beyond the incidence of employer-provided training», Industrial and Labor Relations Review, 52(1), 64-81.

[20] OOSTERBEEK, H. (1996): «A decomposition of training probabilities», Applied Economics, 28, 799-805.

[21] PERAITA, C. (2000): «Características de la formación en la empresa española», Papeles de Economía Española, 86, 295-309.

[22] PERAITA, C. (2005): «Firm sponsored training in regulated labor markets: evidence from Spain», Applied Economics, 37, 1885-1898.

[23] ROYALTY,A.B. (1996): «The effects of job turnover on the training of men and women», Industrial and Labor Relations Review, 49, 506-521

[24] STEVENS, M. (1994): «A theoretical model of on-the-job training with imperfect competition», Oxford Economic Papers, 46, 537-562. 
Apéndice

CUADRO A.1

MODELO LOGÍSTICO DE LA PROBABILIDAD DE QUE UN TRABAJADOR PARTICIPE EN LA FORMACIÓN DE LA EMPRESA

\begin{tabular}{|c|c|c|c|c|c|c|c|c|c|}
\hline & \multicolumn{3}{|c|}{ EPA (1994-2004) } & \multicolumn{3}{|c|}{ ECVT (1999-2004) } & \multicolumn{3}{|c|}{ PHOGUE (1995-2001) } \\
\hline & Coef. & $\mathbf{z}$ & Sig. & Coef. & $\mathbf{Z}$ & Sig. & Coef. & $\mathbf{z}$ & Sig. \\
\hline Sexo (Varón) & & & & & & & & & \\
\hline Mujer & $-0,18$ & $-3,50$ & $* * *$ & $-0,18$ & $-4,41$ & $* * *$ & $-0,10$ & $-1,92$ & \\
\hline Edad (16-24) & & & & & & & & & \\
\hline $25-29$ & $-0,71$ & $-9,96$ & $* * *$ & $-0,09$ & $-1,16$ & & 0,18 & 1,46 & \\
\hline $30-44$ & $-1,16$ & $-16,18$ & $* * *$ & $-0,13$ & $-1,79$ & & 0,28 & 2,39 & ** \\
\hline $45-54$ & $-1,70$ & $-16,13$ & $* * *$ & $-0,13$ & $-1,65$ & & 0,17 & 1,34 & \\
\hline $55-64$ & $-2,44$ & $-13,45$ & $* * *$ & $-0,61$ & $-6,25$ & $* * *$ & $-0,37$ & $-2,37$ & $* *$ \\
\hline Estudios (Obligatorios) & & & & & & & & & \\
\hline Secundarios & 0,40 & 6,12 & $* * *$ & 0,54 & 11,85 & $* * *$ & 0,60 & 8,54 & **** \\
\hline Universitarios & 0,48 & 5,67 & $* * *$ & 0,76 & 13,28 & $* * *$ & 0,82 & 11,38 & $* * *$ \\
\hline Tipo de contrato (Indefinido) & & & & & & & & & \\
\hline Temporal & 0,46 & 6,30 & $* * *$ & $-0,33$ & $-6,32$ & $* * *$ & $-0,29$ & $-3,75$ & $* * *$ \\
\hline Jornada laboral (Parcial) & & & & & & & & & \\
\hline Completa & 0,34 & 6,44 & $* * *$ & 0,29 & 4,13 & $* * *$ & $-0,30$ & $-2,26$ & ** \\
\hline Antigüedad (< 1 año) & & & & & & & & & \\
\hline $1-3$ & 0,28 & 3,83 & $* * *$ & 0,55 & 7,21 & $* * *$ & 0,32 & 3,31 & $* * *$ \\
\hline $4-6$ & 0,20 & 1,88 & & 0,63 & 7,31 & $* * *$ & 0,47 & 4,08 & $* * *$ \\
\hline $7-10$ & 0,21 & 1,94 & & 0,78 & 8,72 & $* * *$ & 0,55 & 4,74 & $* * *$ \\
\hline $11-20$ & 0,24 & 2,39 & ** & 0,78 & 9,08 & $* * *$ & 0,77 & 6,76 & $* * *$ \\
\hline $20+$ & 0,12 & 0,98 & & 0,84 & 9,04 & $* * *$ & 0,67 & 5,86 & $* * *$ \\
\hline Sector (Público) & & & & & & & & & \\
\hline Privado & $-0,84$ & $-10,29$ & $* * *$ & $-0,28$ & $-4,54$ & $* * *$ & $-0,35$ & $-4,51$ & $* * *$ \\
\hline Tamaño (Categoría 1) & & & & & & & & & \\
\hline Categoría 2 & 0,11 & 1,05 & & 0,57 & 9,51 & $* * *$ & 0,33 & 3,52 & $* * *$ \\
\hline Categoría 3 & 0,29 & 3,08 & *** & 0,94 & 14,20 & $* * *$ & 0,49 & 4,93 & $* * *$ \\
\hline Categoría 4 & 0,50 & 6,33 & $* * *$ & 1,06 & 15,44 & $* * *$ & 0,72 & 6,94 & $* * *$ \\
\hline Categoría 5 & $-0,20$ & $-2,31$ & $* *$ & 1,42 & 20,57 & *** & 0,81 & 8,27 & $* * *$ \\
\hline Categoría 6 & - & - & & 1,69 & 20,85 & $* * *$ & 0,76 & 7,83 & $* * *$ \\
\hline Categoría 7 & - & - & & 1,61 & 18,59 & *** & - & - & \\
\hline Categoría 8 & - & - & & 1,55 & 24,78 & $* * *$ & - & - & \\
\hline Rama (Agricultura) & & & & & & & & & \\
\hline Construcción & $-0,26$ & $-1,29$ & & 0,19 & 1,36 & & $-0,25$ & $-1,05$ & \\
\hline Energía, agua y extractivas & 0,29 & 1,08 & & 0,66 & 3,69 & $* * *$ & 0,48 & 1.89 & \\
\hline Transf. metales y química & 0,13 & 0,62 & & 0,55 & 3,83 & $* * *$ & 0,32 & 1,31 & \\
\hline Fabricación de equipo & 0,55 & 2,73 & $* * *$ & 0,71 & 4,65 & $* * *$ & 0,43 & 1,87 & \\
\hline Otras industrias manufactureras & $-0,19$ & $-0,94$ & & 0,26 & 1,84 & & 0,25 & 1,18 & \\
\hline Servicios tradicionales & $-0,01$ & $-0,03$ & & 0,43 & 3,15 & $* * *$ & 0,13 & 0,60 & \\
\hline Servicios productivos & 0,63 & 3,28 & $* * *$ & 0,80 & 5,74 & $* * *$ & 1,22 & 5,41 & $* * *$ \\
\hline Servicios sociales & 0,29 & 1,46 & & 0,57 & 3,98 & $* * *$ & 0,11 & 0,46 & \\
\hline Servicios personales & 0,03 & 0,13 & & 0,21 & 1,26 & & 0,24 & 1,08 & \\
\hline Servicios públicos & $-0,01$ & $-0,03$ & & 0,43 & 2,88 & *** & 0,35 & 1,61 & \\
\hline Ocupación (No manual-cualific.) & & & & & & & & & \\
\hline No manual-poco cualificada & $-0,36$ & $-5,06$ & *** & $-0,34$ & $-6,58$ & $* * *$ & $-0,19$ & $-3,16$ & $* * *$ \\
\hline Manual-cualificada & $-0,21$ & $-2,37$ & $* *$ & $-0,62$ & $-9,54$ & *** & $-0,64$ & -7.06 & $* * *$ \\
\hline Manual-poco cualificada & $-0,93$ & $-9,19$ & $* * *$ & $-0,79$ & $-13,09$ & *** & $-0,87$ & $-9,62$ & *** \\
\hline Constante & $-5,07$ & $-19,98$ & $* * *$ & $-2,74$ & $-13,63$ & $* * *$ & $-3,89$ & $-13,35$ & $* * *$ \\
\hline
\end{tabular}


